\title{
Gentrification: neue Forschungsergebnisse aus Berlin und Köln
}

\author{
Friedrichs, Jürgen; Blasius, Jörg (Hrsg.) (2016): Gentrifizierung in Köln. Soziale, \\ ökonomische, funktionale und symbolische Aufwertungen. Opladen: Verlag Barbara \\ Budrich, 186 S., 41 Abb., 19 Tab. Helbrecht, Ilse (Hrsg.) (2016): Gentrifizierung in \\ Berlin. Verdrängungsprozesse und Bleibestrategien. Bielefeld: Transcript, 322 S., 52 \\ Abb., 13 Tab.
}

\section{Christian Diller ${ }^{1}$}

Eingegangen: 10. Januar 2017 / Angenommen: 13. Februar 2017 / Online publiziert: 1. März 2017

(C) Springer-Verlag Berlin Heidelberg 2017

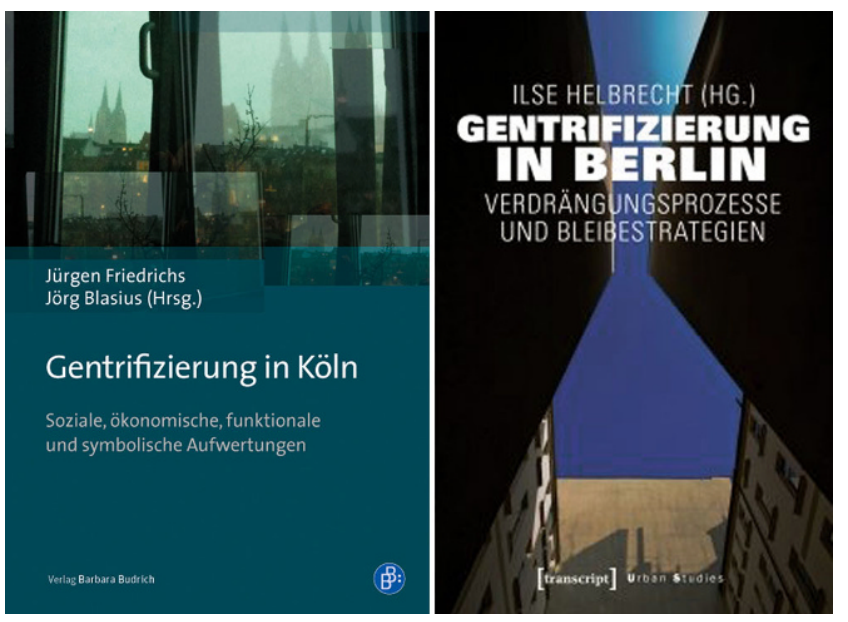

Nur wenige Begriffe der Stadtforschung sind in der Diskussion so aufgeladen wie der Begriff der ,gentrification“, der Gentrifizierung. Inspiriert durch die Debatte in den USA und Großbritannien wird auch in Deutschland bereits seit den 1980er-Jahren zu dem Thema geforscht. Es erlebt derzeit in Deutschland eine Renaissance. Der unübersehbare Trend ,in die Großstädte“ hat vor allem in den deutschen Metropolen zu einer seit einigen Jahren ausgesprochen angespannten Lage auf den Wohnungsmärkten geführt. Eine Reihe von innerstädtischen Quartieren hat bauliche und funktionale Aufwertungen erfahren, somit stellt sich die Frage nach den sozialräumlichen Auswirkungen. Insofern sind die beiden Bände, die sich mit Gentrifizierungsprozessen in Quartieren in der größten und viertgrößten deutschen

Prof. Dr. Christian Diller

christian.diller@geogr.uni-giessen.de

1 Institut für Geographie, Justus-Liebig-Universität Gießen, Senckenbergstraße 1, 35390 Gießen, Deutschland
Stadt befassen, hochaktuell, zumal sie von Herausgebern verantwortet werden, die in der Gentrifizierungsdebatte in Deutschland einschlägig bekannt sind und im Falle von Jürgen Friedrichs und Jörg Blasius bereits die Gentrifizierungsforschung der 1990er-Jahre maßgeblich prägten.

Beiden Bänden ist gemeinsam, dass sie von Teams erstellt wurden, deren Autoren sich auf jeweils bestimmte Aspekte des Themas gentrification konzentrieren. Was die Ressourcen angeht, ist der Hintergrund der Publikationen allerdings verschieden: Während die Gentrifizierungsstudie in Köln in Form eines mehrjährigen, von der FritzThyssen-Stiftung finanzierten Forschungsprojektes und im Kontext eines Projekts der Deutschen Forschungsgemeinschaft (DFG) erstellt wurde, stellt die Berliner Studie das Ergebnis von Master-Modulen im Fach Geographie an der Humboldt-Universität zu Berlin unter der Leitung der Herausgeberin dar. Auch der inhaltliche und der räumliche Fokus der Bände ist unterschiedlich: Während der Band von Jürgen Friedrichs und Jörg Blasius dezidiert von einer holistischen Definition von gentrification ausgeht, die neben der sozialen und baulich-ökonomischen auch die funktionale und symbolische Aufwertung beinhaltet, konzentriert sich der Band von llse Helbrecht auf den sozioökonomischen Wandel und hierbei ausdrücklich auf das Thema der Verdrängten, nach Einschätzung der Herausgeberin ein von Wissenschaft und Politik vernachlässigter Aspekt der Gentrifizierungsforschung. Die wichtigste Leitfrage ist hier: „Wohin ziehen die Verdrängten?“ Umgekehrt ist der Kölner Band räumlich stärker fokussiert als der Berliner Band. Alle seine Beiträge sind systematisch komparativ angelegt: Es geht dabei darum, die Hypothese zu untersuchen, dass die Gentrifizierungsprozesse im Stadtteil Deutz weiter vorangeschritten sind als im Stadtteil Mühlheim.

Ein wichtiges Signal zur Entwicklung des Begriffs gentrification geben Jürgen Friedrichs und Jörg Blasius in 
ihrem einführenden Artikel: die Verknüpfung des etablierten Phasenmodells der Gentrifizierung mit allen ihren ier Dimensionen. Der Invasions-Sukzessions-Zyklus wird mit Hypothesen zu allen Merkmalen der gentrification gefüllt. Das ist insofern bemerkenswert, als zumindest Jürgen Friedrichs in seinen älteren Arbeiten die soziale Dimension zur zentralen erklärt hatte (Friedrichs 1996: 14). Der Nachteil der holistischen Definition, die den Begriff unpräzise macht, wird damit in Kauf genommen, was aber die Anschlussfähigkeit an die allgemeine Gentrifizierungsdebatte erhöht.

Die Beiträge von Mareen Wallasch im Kölner Band und von Christian Döring und Klaus Ulbricht sowie Andrej Holm und Guido Schultz im Berliner Band demonstrieren den hierzulande erreichbaren ,state of the art', sozioökonomische Aufwertung im gesamtstädtischen Maßstab differenziert nach Quartieren zu betrachten, indem Daten der amtlichen Statistik mit Wohnungsmarktdaten (z. B. Immoscout) verknüpft werden. Gerade die Verfügbarkeit von Wohnungsmarktdaten hat sich hier in den letzten Jahren deutlich verbessert. Die Beiträge zeigen, dass auf der Ebene von Aggregatdaten zumindest erste räumliche Gentrifizierungsverdachtsdiagnosen bezogen auf sozioökonomische Aspekte methodisch sauber möglich sind. In Köln verdichten sich die Hinweise, dass die Gentrifizierungswelle den Rhein übersprungen hat, deutlich. Noch beeindruckender ist die Bestätigung des Befundes der „Gentrification-Spirale" für Berlin: Die ökonomische Aufwertung des Wohnungsbestandes scheint nun nach den Stadtteilen Mitte, Prenzlauer Berg, Friedrichshain, Kreuzberg und Neukölln auch Teile Moabits, des Wedding und Gesundbrunnens zumindest in Teilen erfasst zu haben.

Der Berliner Beitrag von Daniel Förste und Matthias Berndt ist insofern methodisch interessant, als sich die $\mathrm{Au}$ toren der Mühen der Wanderungsanalyse zwischen Quartieren unterziehen, indem die Wanderungen aus dem Prenzlauer Berg in die Stadtteile Pankow, Weißensee, Lichtenberg, Hohenschönhausen, Marzahn, Hellersdorf, Friedrichshain, Kreuzberg, Wedding und Neukölln-Nord untersucht werden. Im Ergebnis stützen auch diese Befunde das Modell der Gentrification-Spirale und sprechen gegen die Hypothese eines Wegzugs von sozial schwachen Bevölkerungsgruppen aus innenstadtnahen Gebieten in Berliner Randbezirke. Da jedoch die Sozialstatusgruppen nicht unterschieden werden, lassen sich keine Folgerungen über soziale Selektionsprozesse ziehen, ohne die eine Gentrifizierungsdiagnose nicht möglich ist. Auch hätte die Betrachtung der Zuzüge und der Wanderungssalden zu einem vollständigen Bild gehört.

Den empirisch-methodisch und durch seine Ergebnisse auch theoretisch wohl wichtigsten aller Beiträge stellt der von Jürgen Friedrichs und Jörg Blasius dar, der das etablierte Phasenmodell der gentrification, das inzwischen sogar in
Schulbücher Eingang gefunden hat, überprüft. Die Ressourcen des Forschungsprojekts ermöglichten es, diesen Teil des schon in den 1990er-Jahren von Jürgen Friedrichs (1996: 40) formulierten Forschungsbedarfs zu absolvieren. Dazu wurde ein repräsentatives Panel mit einer repräsentativen Anzahl von Wohnungen (N der Stichprobe: 2.372) in von den Autoren nach Begehungen ausgewählten Teilgebieten der Kölner Stadtteile Mühlheim und Deutz aufgebaut. Mit hohem Aufwand wurde eine ausreichende Zahl von Wohnungen viermal im Abstand von einem Jahr aufgesucht und es wurden Informationen zum sozialen Status der jeweiligen Hauptbewohner erhoben. Das Phasenmodell der gentrification kann dabei nur in Teilen bestätigt werden. Für Mühlheim gibt es sogar Zeitpunkte, zwischen denen der Anteil der "Gentrifier" abnimmt, während der Anteil der Pioniere steigt. Deutlich wird vor allem, dass der Horizont von vier Jahren nicht ausreichend ist, um deutlich erkennbare Phasen des Gentrifizierungszyklus nachzuzeichnen. Fast noch wichtiger ist aber ein anderer Befund: In dem etablierten Phasenmodell der gentrification werden ,Ältere‘ von Anbeginn als geringer werdende Bevölkerungsgruppe angesehen, die ab einem gewissen Punkt zu einer Minderheit in ihren Quartieren wird. In den untersuchten Gebieten sind es jedoch vor allem die, Älteren', deren Anteil am stärksten steigt, in beiden Gebieten in den nun vier beobachteten Jahren von 20 auf 30 Prozent. Anders formuliert: Das wichtigste messbare Merkmal im Wandel der Nachbarschaft in beiden Gebieten ist nicht die ,pionierorientierte Gentrification“, sondern die „Gerontification“. Dieser Befund regt da$\mathrm{zu}$ an, das Phasenmodell der Gentrifizierung mit den darin enthaltenen Akteursgruppen grundsätzlich zu überdenken.

Auch die Untersuchung von Jan Üblacker zu den Kölner Stadtteilen ist vor allem dazu geeignet, einige ungeprüfte Hypothesen und Mythen des Phasenmodells der gentrification zur Rolle der Pioniere in Frage zu stellen; denn ein beträchtlicher Teil der Pioniere nimmt die Veränderungen der Bewohnerstruktur und der Freizeit- und Kulturangebote im Gebiet wahr, ohne sich selbst jedoch als die Gruppe anzusehen, die diese Prozesse durch die vermeintlich ,kreative' Aneignung des Gebietes auch mit ausgelöst hat.

Eine Reihe von Beiträgen aus dem Berliner Band geht der Frage nach den neuen Wohnorten der, Verdrängten auf der Mikroebene - also durch Personenbefragungen nach, dabei werden allerdings durchweg keine generalisierbaren Ergebnisse, sondern bestenfalls erste Hinweise erarbeitet. Simon Koch, Marrike Kortus, Christine Schierbaum und Stephanie Schramm befragten 199 Kreuzberger auf der Straße, ermittelten dabei allerdings nicht tatsächliches Wegzugsverhalten, sondern nur Präferenzen bei einem möglichen Wegzug - eine solche Betrachtung enthält zwangsläufig einen spekulativen Grundtenor. Der Beitrag von Nelly Grotefendt, Malve Jakobsen, Tanja Kohlsdorf und Lina Wegener vertieft das Thema der Wohnsituation sozi- 
al schwacher Bevölkerungsgruppen anhand von Expertenund Betroffeneninterviews. Die Beiträge von Lisa Scheer zu neuen Widerstandsformen gegen Verdrängung und von Paul Neupert zum Leben im Wohnwagen bringen frische Facetten in das Thema, allerdings geht hierbei der Bezug zum Thema Gentrifizierung und entsprechenden Gebieten zum Teil zu sehr verloren.

Mit fortschreitender Lektüre des Berliner Bandes wird jedoch sein Hauptdefizit deutlich: Er beschäftigt sich mit dem Thema „Verdrängung“ als Teil von Gentrifizierung, ohne jedoch eine für alle Beiträge konsistente Definition von Verdrängung geliefert zu haben. Der Einführungsbeitrag der Herausgeberin betont zwar die Wichtigkeit des Themas Verdrängung, setzt sich aber nicht weiter mit dem Begriff auseinander. Im darauffolgenden Beitrag von Christian Döring und Klaus Ulbricht wird zwar eine Definition gegeben, die aber nicht allgemein verbindlich erscheint. Demnach kann von Verdrängung gesprochen werden, wenn Haushalte unfreiwillig aus Gründen umziehen, die durch sie nicht beeinflusst werden können. Die dringend erforderliche differenzierte Auseinandersetzung mit der von Marcuse (1985) entwickelten Typisierung wird jedoch erst im Beitrag von Camilo Betancourt gegen Ende des Buches zumindest angedeutet. Aber auch hier kann der Umgang mit diesem Begriff nicht überzeugen. In den hier geführten Interviews mit Weggezogenen wird vor allem die Vielschichtigkeit von Wegzugsmotiven, individuellen Lebenslagenveränderungen, empfundenen Veränderungen im Gebiet und Befürchtungen der Folgen der Veränderungen seitens der Befragten deutlich. Vor dem Hintergrund einer Interpretation des Begriffs ,,Verdrängung“ als ,unfreiwilliger Wegzug“ ist aus den Analysen jedoch für kein Beispiel nachvollziehbar, warum ein Interviewter als ,Verdrängter“ zu kennzeichnen wäre. Was die Sache weiter erschwert: Der Autor selbst lehnt die Definition von Verdrängung als ,unfreiwilliger Wegzug“" als zu eng ab, ohne freilich eine eigene klare Definition von Verdrängung zu geben. Und auch die Frage, ob die Interviewten sich selbst verdrängt fühlten, wurde bemerkenswerterweise vom Autor den Interviewten bewusst nicht gestellt. Warum eigentlich nicht?

Die Auseinandersetzung mit dem Begriff und den Typen der Verdrängung hätte vermutlich in vielen der Beiträge aus dem Berliner Band $\mathrm{zu}$ einem anderen Vorgehen und zu einer anderen Interpretation der Ergebnisse geführt. So verwenden die Autoren den Begriff ,,Verdrängung“ durchweg zu unreflektiert und im Vergleich der Beiträge auch nicht konsistent. Der einzige Beitrag, der direkte Verdrängung plausibel nachweisen kann, ist der von Greta Ertelt, Charlotta-Elena Schulz, Georg Thieme und Christiane Uhlig. Hier wurden aktuelle und ehemalige Bewohner von drei Häusern befragt, die ehemals im Rahmen des sozialen Wohnungsbaus gefördert und danach privatisiert wurden. Das Ergebnis ist eindeutig: Das Auslaufen der Bindungen hat zu einem deutlichen Bevölkerungsaustausch in den Häusern geführt, der zu einem hohen Anteil aus unfreiwilligen Wegzügen resultierte, da nach der Privatisierung die Mieten sprunghaft gestiegen sind oder gar direkter Druck auf die Mieter ausgeübt wurde. Auch hier versuchten die Weggezogenen, in der Nähe der alten Wohnungen eine neue Wohnung zu finden. So eindrücklich der wichtige Beitrag das Augenmerk auf die Problematik der auslaufenden Mietpreisbindungen im sozialen Wohnungsbau richtet, gelingt es ihm dennoch nicht, die Lücke der Verdrängungs- zur Gentrifizierungsorschung zu schließen. Die Beispiele liegen zwar in Gebieten in Kreuzberg, mehr wird jedoch über die direkten Nachbarschaften nicht bekannt. Hier wäre beispielsweise ein Vergleich der Entwicklungen privatisierter Bestände aus dem sozialen Wohnungsbau in mutmaßlich gentrifizierten Gebieten mit anderen Gebieten aufschlussreich gewesen. Immerhin lassen sich aus der Befragung aber Hinweise für repräsentative und vertiefende Analysen zu dieser wichtigen Frage gewinnen.

Es erscheint paradox, erklärt sich aber sicherlich auch durch die unterschiedlichen Forschungsressourcen der beiden Bände, dass nicht in den Berliner, sondern in den Kölner Studien der einzige Versuch unternommen wurde, Verdrängung aus gentrifizierten Gebieten auch nachvollziehbar nachzuweisen. In Zusammenarbeit mit dem Statistischen Amt der Stadt Köln gelang es immerhin tatsächlich, die Adressen für eine ausreichend große Zahl von Weggezogenen zu erhalten. Leider aber erwies sich die Zahl der Antworten (nur 25\% der 120 weggezogenen Haushalte antworteten) als zu gering für repräsentative Aussagen zu Wegzugsgründen.

Gerade auch der Berliner Band ist jedoch eher ein Beleg dafür, dass die direkte Verdrängung durch gentrification in Deutschland kaum nachweisbar ist. Das hierzulande wesentlich weiter verbreitete und damit auch politisch relevantere Phänomen ist das, was Marcuse (1985: 214) mit dem Begriff des „Exclusionary Displacement“ bezeichnete. Damit ist folgendes Phänomen angesprochen: Eine Mietpartei zieht aus einer Wohnung - aus welchen Gründen auch immer - aus und für die neue Mietpartei wird die Miete so stark erhöht, dass die alte Mietpartei sich diese nunmehr nicht mehr leisten könnte, wenn sie dort wohnen wollen würde. Bezogen auf eine Gesamtstadt bedeutet dies, dass die Wahlmöglichkeiten für eine neue Wohnung für bestimmte Sozialstatusgruppen drastisch eingeschränkt werden. Dieses Phänomen ist in Deutschland unübersehbar, alleine die Preisentwicklungen auf dem Wohnungsmarkt und die Scheren in der Einkommensentwicklung lassen diesen Schluss zu. Aber kann denn dieses Phänomen des „Exclusionary Displacement“, das ja letztlich die Veränderungen auf der Aggregatebene bezeichnet und eben nicht unmittelbar bedeuten muss, dass auf der Mikroebene eine unfreiwillige Umzugsentscheidung getroffen wird, wirklich se- 
riös als „Verdrängung“ bezeichnet werden - zumal dann, wenn sich der Haushalt selbst gar nicht verdrängt fühlt? Handelt es sich bei diesem wichtigen Phänomen also nicht eher um „Exclusionary Replacement“ als um „Exclusionary Displacement"? Stellt sich damit nicht eher die Frage nach Formen und Mechanismen der forcierten räumlichen sozialen Exklusion als der durch Gentrifizierung bedingten Verdrängung?

Genau solche Fragen hätten in dem Berliner Band auf der Basis einer differenzierten, aber dennoch konsistenten Auseinandersetzung mit dem Verdrängungsbegriff behandelt werden müssen. Während der Kölner Band ein durchweg überzeugendes Beispiel für den ,state of the art“ der Gentrifizierungsforschung darstellt, hat der Berliner Band insgesamt experimentellere Züge, macht jedoch vor allem die theoretischen und methodischen Defizite der Verdrängungsforschung und ihre Lücke zur Gentrifizierungsforschung eher deutlich als sie zu schließen. Dennoch ist auch dieser Band, wie die Kölner Studien, ein Muss für alle an dem Thema Interessierten. Er hat vor allem das politisch wichtige Verdienst, zur Differenzierung beizutragen. Für die Berliner Situation geben die Beiträge trotz fehlender Generalisierungsmöglichkeit einerseits sogar eine gewisse Entwarnung: Verdrängung von ehemals zentral Wohnenden in die Randbezirke, was dort zu neuen Problemlagen führen könnte, konnte nicht nennenswert festgestellt werden. Noch wichtiger aber ist, dass der Band eindringlich das Augenmerk auf die Folgen der Wohnungsbaupolitik der letzten 20 Jahre lenkt und den dringenden Handlungsbedarf zur Schaffung preiswerten Wohnraums vor allem in den Metropolen unterstreicht. Dies allerdings ist eine Fragestellung, die mit dem Thema Gentrifizierung zwar Schnittmengen aufweisen mag, aber aus stadtgeographischer Sicht besser in den breiteren Kontext des generellen Wandels von Nachbarschaften und räumlicher Exklusion zu setzen wäre. Dieses Problem ist für sich genommen groß genug und hat den zum Teil abgenutzten Aufmerksamkeitsbegriff gentrification insofern gar nicht nötig.

\section{Literatur}

Friedrichs, J. (1996): Gentrification: Forschungsstand und methodologische Probleme. In: Friedrichs, J.; Kecskes, R. (Hrsg.): Gentrification. Theorie und Forschungsergebnisse. Opladen, 13-40.

Marcuse, P. (1985): Gentrification, Abandonment, and Displacement: Connections, Causes, and Policy Responses in New York City. In: Urban Law Annual. Journal of Urban and Contemporary Law 28, 195-240. 